\title{
Getting Off \\ the Mad Path
}

DEBUGGERS AND

ASSERTIONS

Dear KV,

I just spent the better part of a week debugging a problem in a piece of code that required the programmer-me-to rerun the same 100 lines of code, adding more and more debugging statements until the bug finally revealed itself. Since this particular code was in a serverless environment, a traditional debugger was not an option. Instead, I had to fire off the function, over and over and over and over again. Eventually, my eyes would glaze over and l'd get up, walk around my home office, and sit down to start all over again. I somehow cannot believe that this is the right way to attack a problem like this, but when I asked other members of my team for help, they told me that tedium was to be expected. Clearly that cannot be so, can it?

Teed off at Tedium

\section{Dear Teed,}

So many clichés relate to this topic, I feel I could just quote them all here, make myself a stiff drink, and call it a day. But that seems lazy and unfair, so l'll just make the drink and try to avoid the clichés.

Most of us understand that computers are great at doing the same thing over and over and over and over again. On bad days, $\mathrm{KV}$ feels that this is the only thing that computers are actually good for and that he is trapped inside a surreal, Dr. Seuss nightmare landscape, in which 
strangely drawn, brightly colored characters spew nonsense while causing all my code to crash in ways that are clearly not possible. Unlike some of my colleagues, I don't microdose, so I know that this is my imagination and I can pull the emergency brake on the nightmare and escape at any time without waiting for the dose to wear off.

Debugging code is often iterative, and iterative processes fall into the over-and-over category. You run the function, it gives the wrong output, you scratch your head and think, "Oh, it must be $X$," and you change whatever $X$ is and run it again, and get another wrong result. Then you think, "Oh, it's not $X$, it's $Y$," and you change $X$ back and then try $Y$, run the code again, and... . Down this path lies madness, with or without microdosing, but it is a well-worn path in our industry that we have all walked many times.

There are several strategies for leaving this path of madness.

You've already alluded to the first way off the mad path, and that is to use a debugger. Unfortunately, there are too few good debuggers in the world, and programmers have rarely been inclined to improve this situation, because-as any venture capitalist will tell you-there is very little money to be made in software tooling.

One might think that some of us who work in the industry would work on better debuggers just for our own sanity, but as KV has pointed out before, programmers are optimists: We always think that this time we've got it right and we can write more code rather than debugging what's in front of us now. This is not to say that there are no debuggers; there are, and some of them even work-a few even work well-but those that do are few and far between. 
f the program works well in the debugger, then you can try it without the training wheels and see how it goes.
$\mathrm{KV}$ continues to grind his teeth as he sees code loaded with debugging statements that would be totally unnecessary if the programmers who wrote the code could be both confident in and proficient with their debuggers. If one is lucky enough to have access to a good debugger, one should give extreme thanks to whatever they normally give thanks to and use the damn thing! When $\mathrm{KV}$ was a much younger programmerette, he worked for a boss who insisted that all code be run in the debugger first-and when KV can, that's what he does. If the program works well in the debugger, then you can try it without the training wheels and see how it goes.

Another way off the mad path, and the one that's probably most relevant to your debugger-less environment, is to add some form of assertion to each and every line of the failing function. As the programmer, you certainly should know what the function is supposed to do, so assert that! A good language would let you do this and then let you hide the assertions from view, but I've rarely seen a language do that.

More often, there is an assertion library or 10 of them (because people are so lazy, they won't do a search for what they need before just hacking up their own] that you can use to do this. Do not go down the iterative path to madness by adding assertions only to the things you think are causing the bug. Assert every blasted line in the function and then-if the stars align-you will find the bug on the next run of the code, because you are checking every bit of the function line by line.

Finally, if you are fighting with a function that fails only 
one out of $n$ times, you'll have to take advantage of the fact that computers really are good at doing things over and over, and automate the execution of the failing function so that you can catch the failure. Step up a level, write a loop, make sure it can catch the failure, and then go back-as I said-and make a good, stiff drink. KV is a fan of the Vesper martini, but will attest that if the failure he's looking for is intermittent, he can get through about six of these, and by then, well, debuggery is no longer what's on his mind. Cheers!

Kode Vicious, known to mere mortals as George V. NevilleNeil, works on networking and operating-system code for fun and profit. He also teaches courses on various subjects related to programming. His areas of interest are code spelunking, operating systems, and rewriting your bad code [OK, maybe not that last one]. He earned his bachelor's degree in computer science at Northeastern University in Boston, Massachusetts, and is a member of ACM, the Usenix Association, and IEEE. Neville-Neil is the co-author with Marshall Kirk McKusick and Robert N. M. Watson of The Design and Implementation of the FreeBSD Operating System [second edition]. He is an avid bicyclist and traveler who currently lives in New York City. Copyright $(2021$ held by ownerlauthor. Publication rights licensed to ACM. 